wissen kompakt 2021 · 15:147-148

https://doi.org/10.1007/s11838-021-00137-1

(c) Freier Verband Deutscher Zahnärzte (FVDZ) and Springer Medizin Verlag GmbH, ein Teil von Springer Nature 2021

\section{Vollkeramischer Zahnersatz}

\author{
Marc Schmitter \\ Poliklinik für Zahnärztliche Prothetik, Universitätsklinikum Würzburg, Würzburg, Deutschland
}

\section{Autor}

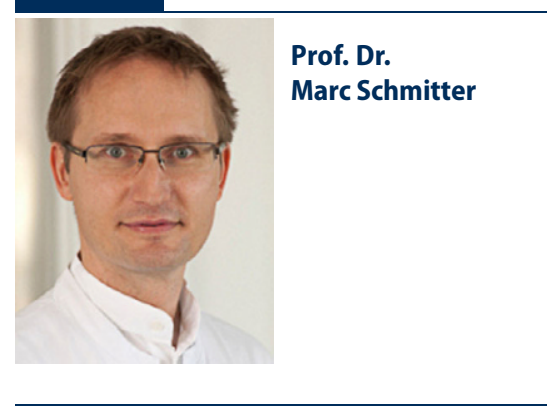

In den letzten beiden Jahrzehnten hat sich bei der Materialauswahl im prothetisch-festsitzenden Bereich viel getan: weg von Kronen und Brücken aus Metalllegierungen hin zu vollkeramischen Werkstoffen. Neben den ästhetischen Vorteilen keramischer Werkstoffe und dem Ausbleiben von Korrosionserscheinungen spielten bei dieser Entwicklung sicherlich auch die fortschreitenden Möglichkeiten der CAD/CAM-basierten Fertigung („,computer-aided design/computer-aided manufacturing") eine maßgebliche Rolle.

Obgleich es bereits vor vielen Jahrzehnten vollkeramische Systeme gab (man denke z. B. an Dicor-Kronen), waren deren materialspezifische Eigenschaften (z.B. Risszähigkeit) nicht optimal, und es waren häufig Misserfolge zu beobachten.

\section{》) Der Siegeszug von vollkerami- schen Restaurationen war anfangs steinig und beschwerlich}

Doch auch nach der Markteinführung von oxidkeramischen Werkstoffen - mit z.B. deutlich besseren Risszähigkeiten - waren vollkeramische Versorgungen oftmals mit gewissen Einschränkungen verbunden: reduzierte ästhetische Ergebnisse bei der Verwendung von Zirkoniumdioxidkeramiken $\left(\mathrm{ZrO}_{2}\right)$, invasive Präparationsrichtlinien, Chippingprobleme bei verblendeten $\mathrm{ZrO}_{2}$-Keramiken, Limitierungen bei der CAD/CAM-gestützten Fertigung, komplexe Befestigungsstrategien usw. Man kann also mit Fug und Recht sagen, dass der Siegeszug von vollkeramischen Restaurationen anfangs steinig und beschwerlich war.

Im Laufe der Jahre wurden die Werkstoffe und deren Fertigungsmöglichkeiten jedoch immer weiter optimiert: 5-AchsFräsmaschinen lösten die 3-Achs-Fräsmaschinen ab, der digitale Workflow konnte erheblich ausgebaut werden, ästhetisch anspruchsvollere $\mathrm{ZrO}_{2}$-Werkstoffe wurden bis zur Marktreife entwickelt, monolithische Restaurationen ersetzten Verbundwerkstoffe wie beispielsweise Verblendkronen usw.

\section{॥V Vollkeramische Restaurationen werden häufig anstelle von metallgestützten Restaurationen eingesetzt}

Diese Entwicklungen sorgten dafür, dass vollkeramische Restaurationen mehr und mehr den Markt erobern und häufig anstellevon metallgestützten Restaurationen bei den Patientinnen und Patienten (erfolgreich) eingesetzt werden. Bezüglich monolithischer $\mathrm{ZrO}_{2}$-Restaurationen bestand jedoch lange Zeit Skepsis. So wurde beispielsweise argumentiert, die Härte des Materials könnte die Antagonisten schädigen und die Kiefergelenke nachhaltig in Mitleidenschaft ziehen. Zwischenzeitlich konnten jedoch viele Studien diese Bedenken deutlich reduzieren und somit den Weg frei für den nahezu ubiquitären Einsatz machen.

In der vorliegenden Ausgabe sollen daher wichtige Eckpunkte beim klinischen Einsatz vollkeramischer Restaurationen etwas näher beleuchtet werden: von der digitalen Abformung über Chancen und Risiken monolithischer Versorgungsvarianten bis hin zur Anwendung im minimalinvasiven Bereich und der adhäsiven Befestigung.

Ich hoffe, diese Beiträge unterstützen Sie bei der erfolgreichen Anwendung vollkeramischer Systeme bei Ihren Patientin- 


\section{Editorial}

nen und Patienten und hoffe, Sie haben genauso viel Freude beim Lesen wie ich.

Mit kollegialen Grüßen

Ihr

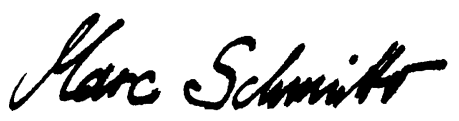

Marc Schmitter

Korrespondenzadresse

Prof. Dr. Marc Schmitter

Poliklinik für Zahnärztliche Prothetik,

Universitätsklinikum Würzburg

Pleicherwall 2, 97070 Würzburg, Deutschland

Schmitter_M@ukw.de

Interessenkonflikt. M. Schmitter gibt an, dass kein Interessenkonflikt besteht. 